\section{A systematic review of the incidence and prevalence of cardiac, cerebrovascular, and peripheral vascular disease in multiple sclerosis}

Multiple Sclerosis Journal

2015, Vol. 21(3) 318-331

DOI: 10.1177

1352458514564485

(C) The Author(s), 2015

\author{
Ruth Ann Marrie, Nadia Reider, Jeffrey Cohen, Olaf Stuve, Maria Trojano, Gary Cutter, \\ Stephen Reingold and Per Soelberg Sorensen
}

Reprints and permissions: http://www.sagepub.co.uk/ journalsPermissions.nav

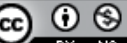

\title{
Abstract
}

Background: Findings regarding the prevalence of vascular comorbidities in multiple sclerosis (MS) are conflicting.

Objective: The objective of this review is to estimate the incidence and prevalence of vascular comorbidities and predisposing comorbidities in persons with MS and to assess the quality of the included studies.

Methods: The PubMed, EMBASE, SCOPUS and Web of Knowledge databases, conference proceedings, and reference lists of retrieved articles were searched. One reviewer abstracted data using a standardized data collection form, while the second reviewer verified the abstraction. Included studies were assessed qualitatively. Quantitatively, we assessed studies using the $I^{2}$ statistic, and conducted meta-analyses for population-based studies only.

Results: The prevalence of hypertension and hyperlipidemia exceeded $10 \%$ in the MS population and increased with age. While the prevalence of ischemic heart disease, congestive heart failure, and stroke were less than $5 \%$ overall, the prevalence of these conditions exceeded expectations when compared to the general population. Cardiac valvular disease, however, affected the MS population less often than expected. Problems with study quality were common.

Conclusion: Despite the relatively high prevalence of some vascular comorbidities in the MS population, important gaps exist in our understanding of their epidemiology. Most of our knowledge is based on studies conducted in a small number of regions.

Keywords: Multiple sclerosis, comorbidity, cardiac, stroke, peripheral vascular disease, incidence, prevalence, systematic review

Date received: 18 August 2014; revised: 23 November 2014; accepted: 25 November 2014

\section{Introduction}

In multiple sclerosis (MS), disease characteristics such as age at symptom onset and type of symptoms at onset are relatively poor at explaining heterogeneity in clinical outcomes such as disability progression. ${ }^{1}$ Several studies suggest that vascular comorbidities including hypertension, hyperlipidemia, and heart disease may adversely influence disability progression. ${ }^{2}$ Hyperlipidemia is associated with the accumulation of new hyperintense lesions on magnetic resonance imaging (MRI). ${ }^{3}$ Further, hypertension and other comorbidities are associated with lower health-related quality of life. ${ }^{4}$

Findings regarding the prevalence of vascular comorbidities in MS are conflicting. For example, some studies have reported that individuals with MS have an increased risk of ischemic heart disease ${ }^{5}$ while others have suggested that individuals with MS are less likely to be hospitalized for ischemic heart disease ${ }^{6}$ Findings regarding stroke also conflict. ${ }^{7-11}$ Therefore, we aimed to systematically review the literature regarding the
Correspondence to: Ruth Ann Marrie University of Manitoba, Health Sciences Center, GF533, 820 Sherbrook Street, Winnipeg, MB R3A 1R9,

Canada.

rmarrie@hsc.mb.ca

Nadia Reider

Department of Internal Medicine, University of Manitoba, Canada

Ruth Ann Marrie Department of Internal Medicine, University of Manitoba, Canada/ Department of Community Health Sciences, University of Manitoba, Canada

Jeffrey Cohen Mellen Center for MS Treatment and Research, Cleveland Clinic, USA

Olaf Stuve

Department of Neurology and Neurotherapeutics,

University of Texas

Southwestern, USA

Maria Trojano

Department of Basic Medical Sciences, Neurosciences and Sense Organs, University of Bari, Italy

Gary Cutter Department of Biostatistics, University of Alabama at Birmingham, USA

Stephen Reingold Scientific and Clinical Review Associates, LLC, USA

Per Soelberg Sorensen Department of Neurology, Copenhagen University Hospital Rigshospitalet, Denmark 
incidence and prevalence of comorbid vascular disorders in MS, and to evaluate the quality of all included studies.

\section{Methods}

This systematic review was part of a study regarding the worldwide incidence and prevalence of comorbidity in MS. Herein we describe the findings for vascular comorbidities including hypertension, hyperlipidemia, cardiac disease, cerebrovascular disease, and peripheral vascular disease.

The approach to developing the search strategies for each comorbidity is delineated in detail elsewhere. ${ }^{12}$ Briefly, we searched the published literature using PubMed and EMBASE, and conference proceedings using SCOPUS and Web of Knowledge for all years available through November 1, 2013 (Supplemental Appendix I). The reference lists of articles identified by electronic searches were manually reviewed.

The abstracts were independently reviewed by two individuals with respect to relevance, with abstracts selected by either reviewer going on to full-text review. To be included a study had to be (i) conducted in an MS population, (ii) clearly identify the comorbidity of interest, (iii) report the incidence or prevalence of the comorbidity, (iv) include original data, and (v) be published in English. Disagreements between reviewers were resolved by consensus.

For each article selected, one reviewer abstracted information using a standardized data collection form. The second reviewer verified the data abstraction. As detailed elsewhere, the data collection form captured general study characteristics, as well as incidence and prevalence estimates. ${ }^{12}$ Each study was critically appraised using a standardized assessment tool used in a systematic review of the incidence and prevalence of MS, and awarded quality scores based on yes or no responses to nine questions. ${ }^{12}$

\section{Statistical analysis}

The $I^{2}$ test was used to quantitatively assess heterogeneity of population-based studies in which the expressed aim was to assess the incidence or prevalence of comorbidity. Random-effects meta-analyses were conducted using a Microsoft Excel spreadsheet developed for this purpose. ${ }^{13} \mathrm{We}$ employed a continuity correction of 0.5 for studies in which zero events were recorded. ${ }^{14}$

\section{Results}

\section{Diabetes}

Search. The search identified 590 unique citations after duplicates were removed (Supplemental Figure 1). After abstract screening and hand searching, 65 articles met the criteria for full-text review, of which we excluded 26, leaving $39.5,7-11,15-47$

Study characteristics. The studies were conducted from 1930 to 2009, but the time period was not reported in several studies. Most studies were conducted in Europe $(19,48.7 \%)$ or North America $(15,38.5 \%)$, with only four (10.2\%) conducted in Asia and two (5.1\%) conducted in Australia. Data sources used included clinical databases or medical records review $(16,41.0 \%)$, administrative data $(12,30.8 \%)$, selfreport $(10,25.6 \%)$, and interviews (seven, 17.9\%); some studies used multiple data sources. Among studies using clinical databases, medical records or interviews, ${ }^{18,19,21,25-27,29,31,36-38,41,42,46,48}$ the diagnostic criteria for MS were reported in nine (56.2\%) studies, ${ }^{25,26,31,33,36-38,40,42,46}$ the Poser criteria were most often used (six, 66.7\%), sometimes in combination with the McDonald criteria. Most studies using administrative data specified the diagnostic codes for MS used; however, only four specified that the administrative case definitions for MS had been validated against a reference standard. ${ }^{5,23,30,34}$ Diabetes was ascertained largely using the same approaches as those used to identify the MS population. Specific diagnostic criteria for diabetes were rarely reported, ${ }^{36,37}$ and the validity of administrative case definitions for diabetes was assessed or described in only three studies..$^{23,30,34}$ Study quality was variable (Supplemental Table 1, associated manuscript ${ }^{12}$ ) with quality scores ranging from one of nine to eight of eight overall, and four of nine to eight of eight among population-based studies.

Incidence. Two studies reported the incidence of diabetes to range from $0.001 \%$ to $1.01 \%$ (Supplemental Table 1). ${ }^{5,47}$

Prevalence. Thirty-nine studies reported prevalence of diabetes, variably for type I diabetes, type II diabetes, or both (Supplemental Table 2)., ${ }^{5-11,15-47}$ Possibly because of the different definitions of diabetes used, and variation in whether the MS cohorts were incident or prevalent, findings varied widely for diabetes (type unspecified) from $0 \%$ to $27.1 \%$. Findings for type I diabetes are discussed with those for autoimmune disease. ${ }^{49}$

For type II diabetes the prevalence varied from $6.75 \%$ to $8.57 \% .8,27$ Only one of these studies was population 
based, reporting the highest estimate. ${ }^{8}$ The prevalence of diabetes appeared to be higher in older populations and in men versus women. The prevalence of diabetes was likely underestimated in the studies relying exclusively on hospital data, $5,7,20,28$ as only hospitalized individuals' data were captured. In the study from New York State only individuals hospitalized for reasons other than MS were included, potentially biasing the estimated prevalence of diabetes. ${ }^{7}$

Seven studies reported diabetes prevalence at or before the diagnosis of MS to range from $0.85 \%$ to $5.65 \% .{ }^{5,17,23,28,30,50,51}$ The prevalence of diabetes reported at MS diagnosis was very similar in the two Scandinavian studies that used similar designs and administrative data sources. ${ }^{5,28}$ These estimates were also quite similar to one based on self-reported diagnoses of diabetes from the North American Research Committee on Multiple Sclerosis (NARCOMS) Registry (1.2\%). ${ }^{50}$

Comparisons. Findings from those studies comparing the prevalence of diabetes in the MS population to another population were completely inconsistent, variously suggesting the prevalence of diabetes to be higher, lower or the same in the MS population. ${ }^{7,8,10,11,17,21,23,25,28,30-34,36,38,42,44-46,51}$ Several of these studies reported comparisons based on the literature, rather than concurrently evaluating a control population (Supplemental Table 3).

\section{Hyperlipidemia}

Search. After duplicates were removed, the search identified 44 unique citations (Supplemental Figure 2). After abstract screening and hand searching, 18 articles met the criteria for full-text review, of which we excluded five, leaving 13 (Supplemental Table 4). ${ }^{7-10,16,26,29,34,35,39,40,44,51}$

Study characteristics. The studies were conducted from 1984 to 2010. Most were conducted in North America (eight, 61.5\%), while three (23.1\%) were conducted in Asia (all in Taiwan), one (7.7\%) study was conducted in Europe and one (7.7\%) was conducted in Australia. Diagnoses of MS and hyperlipidemia were established by several methods including administrative data either with or without formal validation of the case definition for MS (six, $46.1 \%$ ), self-report (four, 30.8\%), and review of medical records or clinical database (three, $23.0 \%$ ). Only one of the studies that used administrative data reported efforts to validate the approach used to identify hyperlipidemia. ${ }^{34}$ One of the studies from Taiwan aimed to improve the specificity of hyperlipidemia as identified using administrative data by requiring one hospital or two ambulatory claims for hyperlipidemia over two years, but the accuracy of this approach was not reported. ${ }^{8}$ Study quality was highly variable, with quality scores ranging from two of nine to eight of eight overall and from four of eight to eight of eight among population-based studies (Supplemental Table 2, accompanying manuscript $\left.{ }^{12}\right)$.

Incidence. None of the studies reported the incidence of hyperlipidemia.

Prevalence. The prevalence of hyperlipidemia ranged from $3.0 \%$ to $47.8 \%$. In the study from New York State only individuals hospitalized for reasons other than MS were included, potentially biasing the estimated prevalence of hyperlipidemia. ${ }^{7}$ The prevalence of hyperlipidemia was particularly high among a population of male veterans at $48.5 \%$ (Supplemental Table 4). ${ }^{10}$

We identified only three population-based studies that aimed to estimate the prevalence of hyperlipidemia; 8,3439 all used administrative data. The first used a validated case definition and reported a prevalence of $13.8 \%(12.4 \%-15.2 \%)$ in $2005,{ }^{34}$ but noted a rising prevalence of hyperlipidemia over time. Heterogeneity was high, as measured by the $I^{2}$ statistic (94.9). The summary prevalence estimate for these studies was $10.9 \%$ (95\% confidence interval (CI): $5.6 \%-16.1 \%$ ) (Figure 1).

Comparisons. Of the seven studies that compared the prevalence of hyperlipidemia in the MS population with a concurrent control population or published data for the general population, five reported a higher prevalence of hyperlipidemia in the MS population (Supplemental Table 5)..$^{8-10,44,51}$ Three of these studies used the same administrative data source in Taiwan. ${ }^{8,44,51}$ The other two studies reported a lower prevalence, ${ }^{7,34}$ but one reported that although hyperlipidemia was less prevalent in the MS population over a 20 -year period, this disparity had dissipated by 2005 (Supplemental Table 5). ${ }^{34}$

\section{Hypertension}

Search. The search identified 218 unique citations (Supplemental Figure 3). After abstract screening and hand searching, 26 articles met the criteria for fulltext review, of which we excluded six. Twenty studies were the subject of this review.

Study characteristics. The studies were conducted from 1997 to 2009.4,5,7-11,16,26,28,32-35,40,44,51-54 Most 


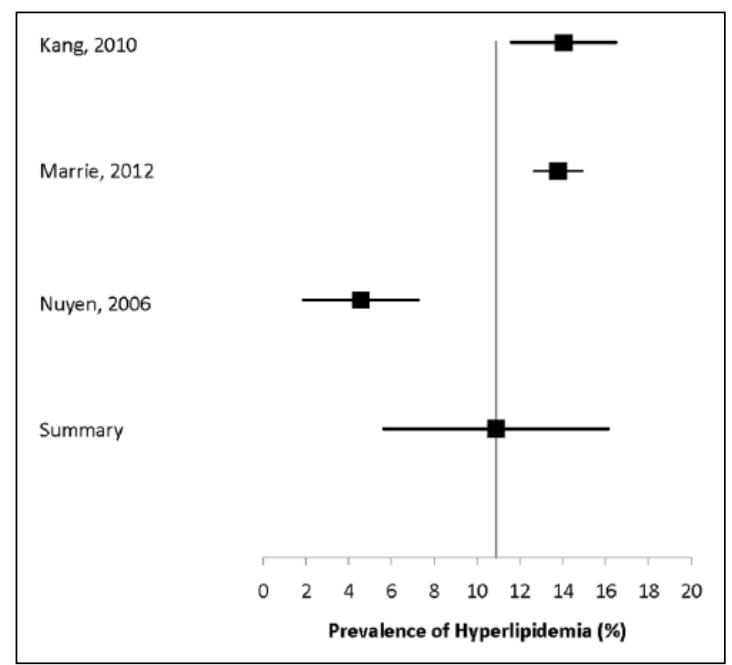

Figure 1. Forest plot of the prevalence of hyperlipidemia in multiple sclerosis in population-based studies.

were conducted in North America (10,50\%), while three $(15 \%)$ were conducted in Europe and the other two $(10 \%)$ were conducted in Asia (Taiwan). Diagnoses of MS were established by several methods including administrative data either with or without formal validation of the case definition for MS (nine, $45 \%$ ), self-report (three by questionnaire or interview, $15 \%$ ), and review of medical records or clinical database (three, 15\%). Hypertension was ascertained by administrative data codes (nine, 45\%), self-report (five, $25 \%$ ), and review of medical records or a clinical database (one, 5\%). Only one of the studies that used administrative data reported efforts to validate the approach used to identify hypertension. ${ }^{34}$ One of the studies from Taiwan aimed to improve the specificity of hypertension as identified using administrative data by requiring one hospital or two ambulatory claims for hypertension over two years, similar to a validated Canadian definition, ${ }^{55}$ but the accuracy of this approach was not reported. ${ }^{8}$ Study quality was highly variable, with quality scores ranging from two of seven to eight of eight overall, and from four of nine to eight of eight among population-based studies (Supplemental Table 2, associated manuscript ${ }^{12}$ ).

Incidence. The only study reporting the incidence of hypertension found that $3.73 \%$ of an incident MS cohort developed the condition over a maximum follow-up of 30 years (Supplemental Table 6). ${ }^{5}$

Prevalence. The reported prevalence of hypertension varied substantially from $0 \%$ to $47.8 \%, 4,5,7-11,16,26,28,32-$ $35,40,44,51-54$ but the study populations were heterogeneous, including both incident and prevalent MS

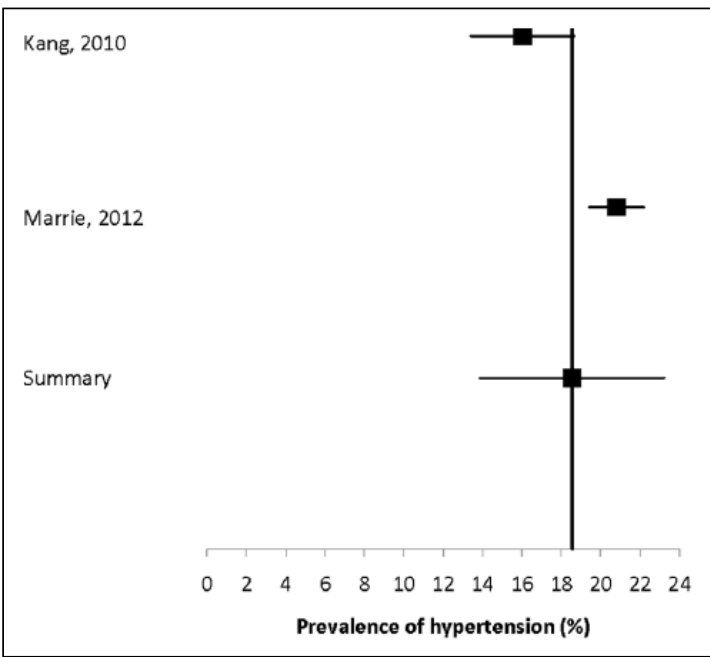

Figure 2. Forest plot of the prevalence of hypertension in multiple sclerosis in population-based studies.

cohorts, and cohorts restricted to men. Some studies evaluated the prevalence of hypertension at or before the diagnosis of MS while others evaluated it after MS diagnosis (Supplemental Table 7). The prevalence of hypertension was likely biased in the studies relying exclusively on hospital data, as only hospitalized individuals were captured. In the two truly populationbased studies with the aim of evaluating the prevalence of hypertension, the findings varied from $16 \%$ to $20.8 \%{ }^{8,34}$ Both studies used administrative data, with reasonably similar case definitions for hypertension. Heterogeneity was high, as measured by the $I^{2}$ statistic (89.9). The summary prevalence estimate for these studies was $18.6 \%$ (95\% CI: 13.9\%-23.2\%) (Figure 2).

In four studies, the prevalence of hypertension at diagnosis ranged from $0 \%$ to $8 \%, 5,28,35,51$ with the highest reported prevalence being in the North American cohort. ${ }^{35}$

Comparisons. Two Scandinavian studies reported the incidence of hypertension in incident MS populations and in the general population but did not make statistical comparisons. ${ }^{5,28}$ Eleven studies compared the prevalence of hypertension in the MS population with a comparator population. ${ }^{7-11,32-34,44,51}$ Six of these reported that hypertension was less common in the MS population, $8,10,33,44,51$ while the others reported that it was more common (Supplemental Table 8). ${ }^{8,10,33,44,52}$

\section{Cardiac arrhythmia}

Search. The search identified 41 citations (Supplemental Figure 4). After abstract screening and hand 


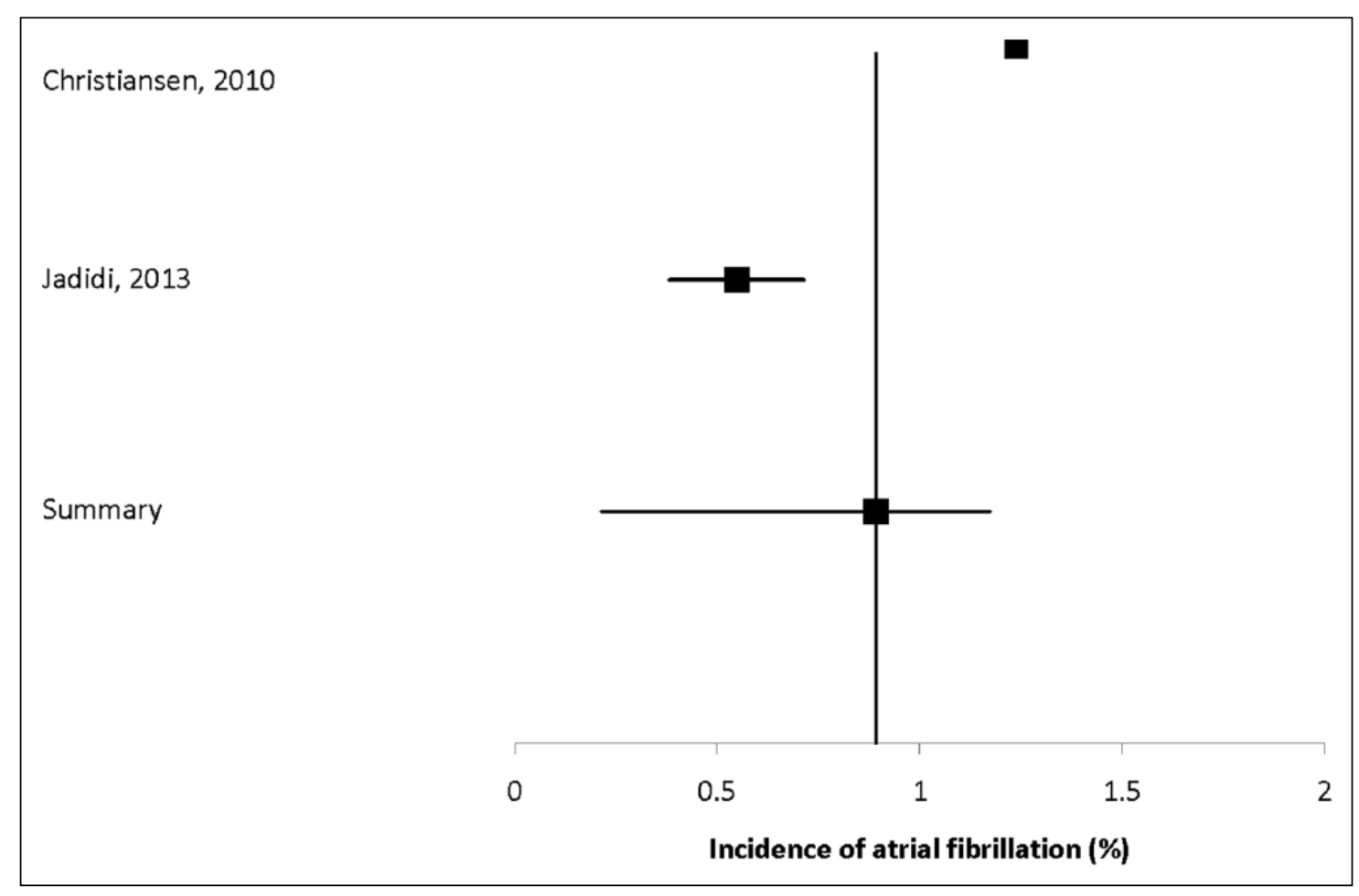

Figure 3. Forest plot of the incidence of atrial fibrillation in multiple sclerosis in population-based studies.

searching, five articles met the criteria for full-text review, of which we excluded one. Four studies were the subject of this review. $5,8,11,28$

Study characteristics. The studies were conducted from 1977 to 2009 . Half of the studies were conducted in Europe, ${ }^{5,28}$ while the other two were conducted in North America and Asia. ${ }^{8,11}$ All of these studies used administrative data and identified the MS population and comorbidities of interest using International Classification of Diseases (ICD) codes. While one study cited high predictive values for diagnoses based on hospital administrative data, ${ }^{28}$ validity of the administrative codes used was generally not reported. Study quality was variable (Supplemental Table 2, associated manuscript ${ }^{12}$ ) with quality scores ranging from four of eight to six of eight overall and from four of eight to six of eight among the three population-based studies. ${ }^{5,8,28}$ Limitations of the studies included failure to report validity of the administrative case definitions used to identify the MS population or cardiac arrhythmia, and failure to age-standardize findings. For example, age-standardized findings were reported in only one study. ${ }^{28}$

Incidence. Both European studies reported the incidence of atrial fibrillation or flutter in incident MS cohorts. 5,28 The incidence was $1.2 \%$ in Denmark and $0.55 \%$ in Sweden (Supplemental Table 9). ${ }^{5,28}$ Since these studies relied on inpatient hospitalizations, the incidence of atrial fibrillation may have been underestimated slightly. Based on an $I^{2}$ statistic of $96.6 \%$, heterogeneity was high. The summary estimate of the incidence of atrial fibrillation was $0.89 \%(95 \% \mathrm{CI}$ : $0.68 \%-1.11 \%$ ) (Figure 3).

Prevalence. Two studies reported the prevalence of cardiac arrhythmia, with estimates ranging from $2.6 \%$ to $4.5 \%$ (Supplemental Table 10). ${ }^{8,11}$ Only one of these studies was population based.

Comparisons. All studies compared the burden of cardiac arrhythmias in the MS population with the general population, but findings conflicted. The two European studies found the incidence of atrial fibrillation was about one-third lower in the incident MS population than in the matched population. ${ }^{5,28}$ Similarly the prevalence of atrial fibrillation was lower in the hospitalized elderly population with MS than in the non-MS hospitalized population. ${ }^{11}$ However, in the Taiwanese population, which largely consists of ethnic Chinese, cardiac arrhythmia occurred more than twice as often in the MS population as in the matched population (Supplemental Table 11). ${ }^{8}$ 
Cardiac valvular disease

Search. The search identified two unique citations (Supplemental Figure 5). After abstract screening and hand searching, four articles met the criteria for fulltext review, of which we excluded two. Two studies were the subject of this review. 5,28

Study characteristics. The studies were conducted from 1977 to 2009. Both studies were conducted in Europe and used incident MS cohorts, as identified using diagnoses in inpatient hospital registers (administrative data). The Danish study cited the positive predictive value of a diagnosis of MS in the hospital register although the citation referred to an abstract, ${ }^{5}$ but the Swedish study did not report the validity of MS as identified using administrative data. Neither study reported the validity of diagnoses of valvular disease based on administrative data. Quality scores ranged from five of eight to six of eight (both population based).

Incidence. Incidence of cardiac valvular disease was reported by one study to be $0.7 \%$ over a 30 -year follow-up period (Supplemental Table 12). ${ }^{5}$

Prevalence. Both studies reported the prevalence of cardiac valvular disease at the time of first diagnosis of MS to range from $0 \%$ to $2.3 \%$ (Supplemental Table 13). ${ }^{5,28}$ The sex distributions of the two populations were similar (64.1\% and $68.05 \%$ female). Because of differences in the way the age distributions were reported they were difficult to compare, but the Danish study reported that $3.8 \%$ of the population was over age 70 years, ${ }^{5}$ while $13.6 \%$ of the Swedish study population was over age 60 years. ${ }^{28}$ The relatively high proportion of older individuals in both studies suggests that some of the MS cases identified were not truly incident. Since valvular disease was identified in only hospitalized individuals the true prevalence may be underestimated. Neither of these studies investigated valvular disease as the primary aim.

Comparisons. Neither study reported a statistical comparison of the prevalence of valvular disease between the MS population and controls, but valvular disease consistently occurred less often in the MS population (Supplemental Table 14).

\section{Ischemic heart disease}

Search. The search identified 170 unique citations (Supplemental Figure 6). After abstract screening and hand searching, 19 articles met the criteria for full-text review, of which we excluded five. Fourteen studies were the subject of this review. $5,7-11,23,26,28,29,32,39,44,56$
Study characteristics. The studies were conducted from 1970 to 2009. Most studies were conducted in North America (six, $42.8 \%$ ) or Europe (five, $35.7 \%$ ), with only two $(14.3 \%)$ conducted in Asia and one (7.1\%) conducted in Australia. Diagnoses of MS were established by several methods including administrative data either with or without formal validation of the case definition for MS (nine, 64.3\%), self-report (two by questionnaire or interview, 14.3\%), and diagnoses in an electronic health record (one, $7.1 \%$ ) or clinical database (one, 7.1\%). Ischemic heart disease was ascertained by administrative data codes (nine, $64.3 \%$ ), self-report (three, $21.4 \%$ ), and review of medical records, electronic charts or a clinical database (three, 21.4\%). With one exception, studies that used administrative data did not report the validity of the case definitions used for ischemic heart disease. ${ }^{56}$ Quality scores ranged from three of seven to eight of eight overall and from four of eight to eight of eight among population-based studies (Supplemental Table 2 , associated manuscript ${ }^{12}$ ).

Incidence. Three studies, two from Europe and one from North America, reported the incidence of ischemic heart disease. ${ }^{5,28,56}$ The two European studies reported that the incidence of myocardial infarction in incident MS cohorts ranged from to $2.36 \%$ to $2.75 \%$ (Supplemental Table 15).5,28 The North American study reported the incidence of ischemic heart disease, not restricted solely to myocardial infarction, but also in an incident MS cohort. ${ }^{56}$

Prevalence. Ten studies reported the prevalence of ischemic heart disease, while one reported the prevalence of cardiovascular disease, without distinguishing ischemic heart disease specifically (Supplemental Table 16). ${ }^{7-11,23,26,29,32,39,44,56}$ The crude prevalence of ischemic heart disease ranged from $0.78 \%$ to $22.2 \%$. The crude prevalence of myocardial infarction was $1.43 \%$ in a contemporary American cohort. ${ }^{7}$ In a temporally more remote Swedish cohort the prevalence of myocardial infarction was as high as $12.7 \%$ in older males. ${ }^{32}$ The prevalence of ischemic heart disease was lower in incident MS cohorts than prevalent MS cohorts.

Among three population-based studies that aimed to estimate prevalence of ischemic heart disease, ${ }^{8,39,56}$ heterogeneity was high $\left(I^{2}=97.6 \%\right)$. The summary estimate of prevalence was $2.50 \%$ (95\% CI: $0-5.77 \%$ ) (Figure 4).

Comparisons. All of the studies that reported the incidence of ischemic heart disease also compared findings to matched populations (Supplemental Table 


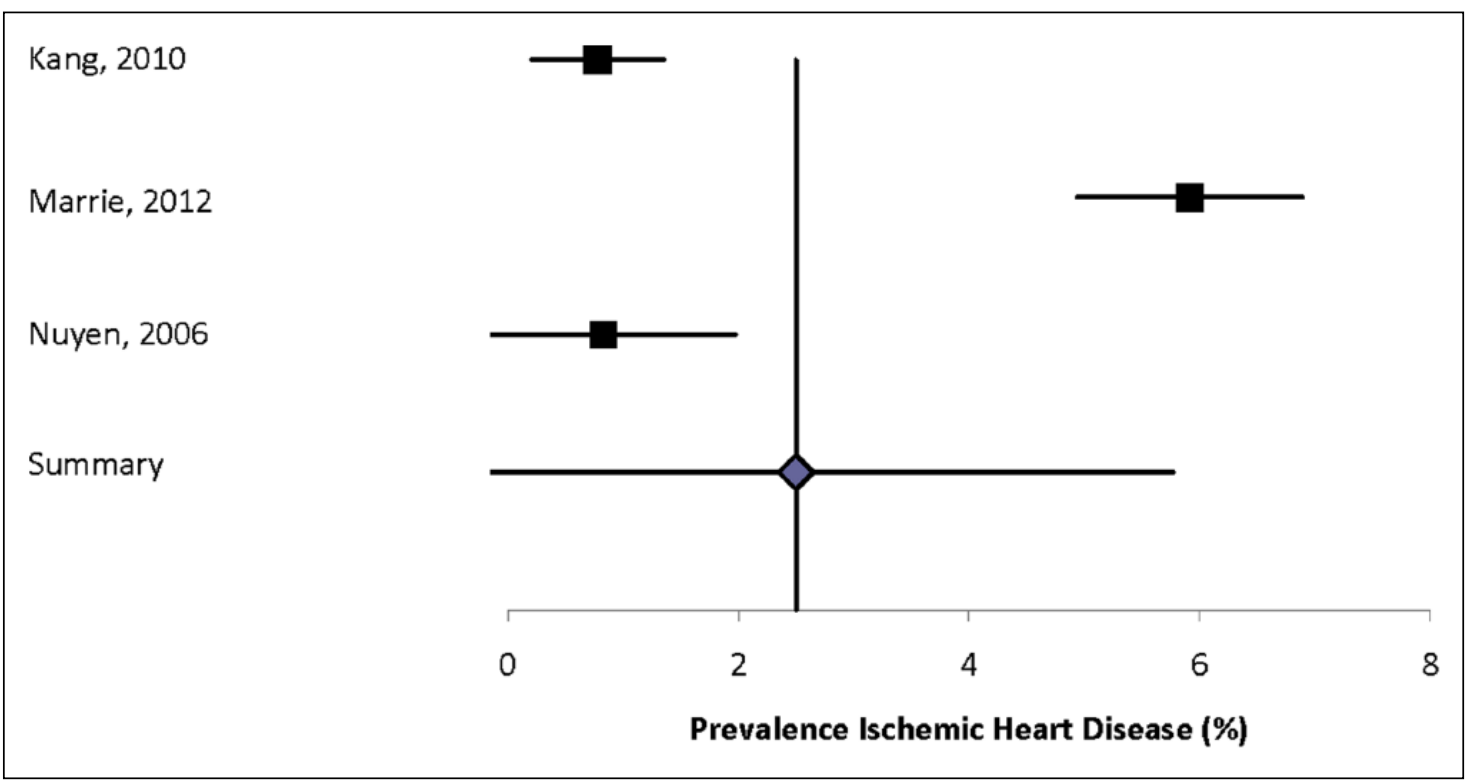

Figure 4. Forest plot of the prevalence of ischemic heart disease in multiple sclerosis in population-based studies.

$17) ; 5,28,56$ all found elevated incidence rate ratios in their incident MS populations.

\section{Congestive heart failure (CHF)}

Search. After duplicates were removed the search identified 421 abstracts (Supplemental Figure 7). After abstract screening and hand searching of reference lists, 10 articles met the criteria for full-text review, of which we excluded six, leaving four. ${ }^{5,8,11,28}$

Study characteristics. The time periods covered by the studies ranged from 1977 to 2009. Half of the studies were conducted in Europe, both in Scandinavian countries, ${ }^{5,28}$ while the others were conducted in North America ${ }^{11}$ and Asia. ${ }^{8}$ All of the studies used administrative data.

Quality scores ranged from four of eight to seven of eight (all population-based studies) (Supplemental Table 18). Limitations of the studies included failure to report validity of the administrative case definitions used to identify the MS population or CHF, focus on hospital data rather than hospital and outpatient data, and failure to age-standardize findings. Christiansen et al. reported that the positive predictive value of MS in their source database was $94 \% .^{5}$ The other studies did not report on the validity of their case definitions for MS, and none validated their administrative case definitions for $\mathrm{CHF}$ against a reference standard.
Incidence. Two European studies reported the incidence of CHF in incident MS cohorts, with findings ranging from $0.89 \%$ to $2.86 \%$ (Supplemental Table 19). $.5 \%$ Heterogeneity of the findings was high $\left(I^{2}=\right.$ $99.2 \%)$. The summary estimate of incidence was $1.87 \%$ (95\% CI: $0 \%-3.80 \%)$.

Prevalence. Two studies, one population-based, reported the prevalence of $\mathrm{CHF}$, with findings ranging from $1.8 \%$ to $5.39 \%$ (Supplemental Table 20). ${ }^{8,11}$ The prevalence of CHF was higher in the American study in which all members of the MS population were hospitalized, and aged 65 years and older. ${ }^{11}$

Comparisons. All of the studies compared the incidence or prevalence of CHF in the MS population to a comparison population (Supplemental Table 21). The Danish study found a nearly two-fold increased incidence of CHF in the incident MS population versus an age- and sex-matched cohort from the general population in the first year after MS diagnosis, ${ }^{5}$ but not over a 30-year follow-up period. The Swedish study also found a nearly two-fold increased risk of CHF in the MS population as compared to an age- and sex-matched cohort from the general population. ${ }^{28}$ The Taiwanese study comparing the prevalence of CHF in a prevalent MS population as compared to matched controls similarly found a two-fold increased risk of CHF in the MS population. ${ }^{8}$ However, the North American study, which was restricted to hospitalized patients with prevalent MS over age 65 years, 
drew the opposite conclusion, possibly because of differences in study population and design. ${ }^{11}$

\section{Cerebrovascular disease}

Search. The search identified 515 abstracts for relevance review (Supplemental Figure 8). After abstract screening and hand searching of reference lists, 11 articles met the criteria for full-text review, of which we excluded one. Ten studies were the subject of this review. . $^{5-11,22,28,39,57}$

Study characteristics. The studies were conducted from 1977 to 2010 . Four (40\%) of the studies were conducted in Europe, while the others were conducted in North America (four, 40\%) and Asia (one, 10\%) and Australia (one, 10\%). Eight of these studies used administrative data and identified the MS population and outcomes of interest using ICD codes. One study cited a high predictive value for MS diagnoses based on hospital administrative data; ${ }^{5}$ validity of the administrative codes used was generally not reported. Three studies were population based. Limitations of the studies included failure to report validity of the administrative case definitions used to identify the MS population or stroke, and failure to age-standardize findings. The remaining studies used self-report to identify comorbid cerebrovascular disease. Quality scores ranged from two of nine to six of eight overall, and from four of eight to six of eight among population-based studies.

Incidence. Two Scandinavian (Denmark, Sweden) studies using administrative data reported the incidence of stroke, both in hospitalized, incident MS populations (Supplemental Table 22). ${ }^{5,28}$ The duration of follow-up and case definitions for stroke differed slightly between studies, but the findings were similar. The incidence of any stroke was $2.85 \%$ in Denmark, ${ }^{5}$ and $2.53 \%$ in Sweden. ${ }^{28}$ The reported incidence of ischemic stroke in Denmark was $1.22 \%$. Heterogeneity of these studies was moderate $\left(I^{2}=47.4\right)$. The summary estimate for the incidence of any stroke was $2.73 \%$ (95\% CI: $2.51 \%-2.95 \%$ ) (Figure 5).

Prevalence. Eight studies reported the prevalence of cerebrovascular disease, with one of the studies being restricted to men with MS (Supplemental Table 22). ${ }^{7-}$ 11,22,39,57 The definition of stroke varied, in some cases simply being any type of stroke, and in others divided into ischemic and hemorrhagic stroke. The prevalence of any stroke varied from $0.4 \%$ to $7.0 \%$, while the prevalence of ischemic stroke varied from $1.2 \%$ to $1.4 \%$. Only one study presented sex-specific data for both sexes, and observed a higher prevalence of stroke in women. ${ }^{57}$ The two population-based studies aimed to estimate prevalence of stroke, with findings ranging from $0.4 \%$ to $6.2 \% .{ }^{8,39}$ The prevalence of stroke was higher in the Taiwanese study, which consisted largely of ethnic Chinese, than in the Dutch study with a predominantly Caucasian population. Heterogeneity of the estimates was substantial $\left(I^{2}=97.4\right)$. The summary estimate was 3.28\% (95\% CI: $0 \%-$ 8.98\%) (Supplemental Figure 9).

Comparisons. Seven studies compared the incidence (two) or prevalence (five) of stroke in the MS and a comparator population (Supplemental Table 23). In both studies the incidence of any stroke and ischemic stroke was greater in the MS population than in matched populations. ${ }^{5,28}$ The prevalence of any stroke and ischemic stroke was higher in the MS population than in the comparator populations in four studies, ${ }^{7-10}$ but lower in the fifth study. ${ }^{11}$ The prevalence of hemorrhagic stroke did not differ between the hospitalized MS population and matched controls in the single study that evaluated this question. ${ }^{7}$

\section{Peripheral vascular disease}

Search. The search identified 30 abstracts for relevance review (Supplemental Figure 10). After abstract screening and hand searching, three articles met the criteria for full-text review, all of which were included (Supplementary Table 24). ${ }^{8,35,40}$

Study characteristics. The studies were conducted from 2000 to 2007, two (66.6\%) in North American and one (33.3\%) in Asia. The methods used in the studies differed substantially, variously using administrative data without validation of the diagnostic codes or algorithm used, self-report using a validated questionnaire, and medical records review to establish the MS study populations and determine the prevalence of administrative data. Study quality was variable with quality scores ranging from four of eight to six of eight (same variability among populationbased studies). Limitations of some of the studies included not being population based, failure to report validity of the approaches used to identify the MS population or peripheral vascular disease, and failure to age-standardize findings.

Incidence. None of the studies reported the incidence of peripheral vascular disease.

Prevalence. In all three of the studies the prevalence of peripheral vascular disease was low, ranging from $1.0 \%$ to $3.8 \%$ (Supplemental Table 24). $8,35,40$ The prevalence of peripheral vascular disease at MS 


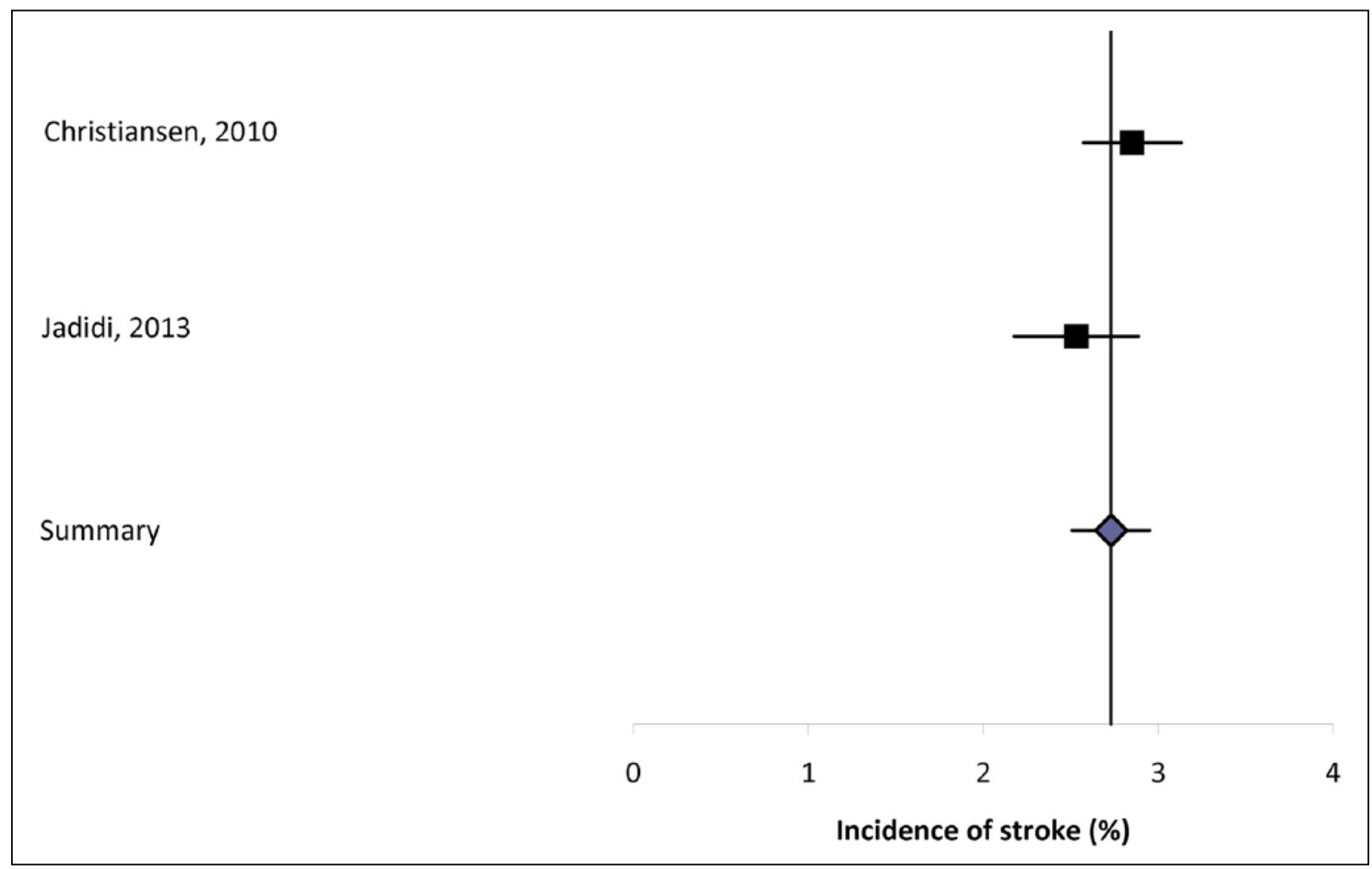

Figure 5. Forest plot of the incidence of stroke in multiple sclerosis in population-based studies.

diagnosis was even lower $(0.41 \%)$ in the only study that evaluated this question. ${ }^{50}$ Heterogeneity among the two population-based estimates was high $\left(I^{2}=\right.$ $88.2 \%)$. The summary estimate for these studies was $2.40 \%$ (95\% CI: $0 \%-5.14 \%)$.

Comparisons. Two of the studies compared the prevalence of peripheral vascular disease in the MS and general populations (Supplemental Table 25). ${ }^{8,58}$ The population-based Taiwanese study observed that the prevalence of peripheral vascular disease was six-fold higher in the MS population than in matched controls. ${ }^{8}$ The study using the NARCOMS volunteer population compared the findings to those obtained from the National Health and Nutrition Examination Survey (NHANES) population. ${ }^{58}$ No clear conclusions could be drawn because of differences in the way the findings were reported with respect to age.

\section{Discussion}

Worldwide, the burden associated with cardiovascular disease and cerebrovascular disease is considerable, but incidence, prevalence, and associated mortality rates vary substantially by region. ${ }^{59-61} \mathrm{We}$ identified relatively few population-based studies that evaluated the epidemiology of comorbid vascular disease in MS. Based on these studies the prevalence of ischemic heart disease and stroke was less than 5\%; however, the prevalence of these conditions increased with age. Given the paucity of studies in regions other than North America and Europe (largely Scandinavia), variations in study design, and lack of standardization of the estimates to account for differences in the age and sex structure of the underlying populations, we could not evaluate geographic variation in the incidence or prevalence of these comorbidities in the MS population. This is an important issue that can be addressed only by conducting similarly designed studies concurrently in multiple regions. To further minimize heterogeneity due to methodologic issues, any such studies using administrative data should also address how well comorbidities are coded in different jurisdictions as under-reporting may occur, ${ }^{62}$ potentially differentially.

Based on this systematic review, hypertension, hyperlipidemia, and diabetes are reasonably common in the MS population. The prevalence is higher in prevalent MS populations than in incident MS populations, increases with age, and is higher among men than women. Because of the age-associated increases in disease prevalence, we can expect that these conditions are more common in individuals with longer disease duration, and may be a particular concern as very few studies explicitly aimed to evaluate the incidence of these conditions, and age- and sex-specific estimates were limited. This particularly limits the ability to assess the impact of shorter survival in the 
MS population on assessment of the incidence and prevalence of conditions that are most prevalent at older ages, such as these ones. These conditions are sufficiently frequent, and associated with enough morbidity in the MS and general population, ${ }^{2,3}$ to warrant specific efforts at prevention.

Although the findings regarding the incidence and prevalence of cardiovascular and cerebrovascular disease were variable, most well-designed studies suggested an increased risk of ischemic heart disease, CHF, ischemic stroke, and peripheral vascular disease in the MS population as compared to the general population. This may reflect several factors. First, individuals with MS have a higher prevalence of smoking, overweight, and obesity than the general population. Second, they have relatively low levels of physical activity, even early in the disease course when disability is mild. These lower levels of physical activity are associated with subclinical atherosclerosis. ${ }^{63}$ The studies we reviewed suggest that diabetes, hypertension, and hyperlipidemia are reasonably prevalent, with a particularly high burden in some populations, although not increased as compared to the general population. ${ }^{10,34}$ Finally, in other immune-mediated conditions such as rheumatoid arthritis and psoriasis, ${ }^{64,65}$ the risk of vascular disease is not fully explained by these traditional risk factors, suggesting that inflammatory processes related to the conditions themselves independently increase the risk of disease.

The prevalence of cardiac arrhythmias and valvular disease, which have been the subject of very limited work, were less than $5 \%$. Unexpectedly, the prevalence of valvular disease was lower than expected when compared to age- and sex-matched populations. Risk factors for acquired valvular heart disease include increasing age, hypertension and hyperlipidemia, smoking, overweight or obesity, physical inactivity, rheumatic fever, as well as risk factors for endocarditis. ${ }^{66}$ With the exception of risk factors for endocarditis, mainly intravenous drug use, which has been little studied in MS, these other risk factors appear to affect the MS population with equal or greater frequency to the general population. Therefore differences in the prevalence of valvular disease might reflect less adequate control of risk factors in the MS population, or differences in disease ascertainment. This requires further evaluation.

Given prior work reporting an increased risk of death from cardiovascular disease, ${ }^{67}$ and adverse impacts on disability progression, an increased focus on these comorbidities in the MS population is required. Studies to establish the incidence and prevalence of these conditions in the MS population worldwide are needed. It is important to understand the clinical characteristics of vascular comorbidities in the MS population, the role of common risk factors. and the role of novel disease-modifying therapies in determining the risk of these conditions. Future research should also focus on understanding the influence of treatments used for these comorbidities on MS. ${ }^{68}$

\section{Acknowledgements}

Thanks to Tania Gottschalk, BA, MEd, MSc (Librarian, University of Manitoba), who provided assistance regarding the development of the search strategies for this review. This study was conducted under the auspices of the International Advisory Committee on Clinical Trials of New Drugs in Multiple Sclerosis, whose members include Jeffrey Cohen, MD (Cleveland Clinic Foundation, Cleveland, United States), Laura J. Balcer, MD, MSCE (NYU Langone Medical Center, New York City, United States), Brenda Banwell, MD (The Children's Hospital of Philadelphia, Philadelphia, United States), Michel Clanet, MD (Federation de Neurologie, Toulouse, France), Giancarlo Comi, MD (University Vita-Salute San Raffaele, Milan, Italy), Gary R. Cutter, PhD (University of Alabama at Birmingham, Birmingham, United States), Andrew D. Goodman, MD (University of Rochester Medical Center, Rochester, United States), Hans-Peter Hartung, MD (Heinrich-Heine-University, Duesseldorf, DE), Bernhard Hemmer, MD (Technical University of Munich, Munich, Germany), Catherine Lubetzki, MD, $\mathrm{PhD}$ (Fédération des maladies du système nerveux et INSERM 71, Paris, France), Fred D. Lublin, MD (Mount Sinai School of Medicine, New York, United States), Ruth Ann Marrie, MD, PhD (Health Sciences Centre, Winnipeg, Canada), Aaron Miller, MD (Mount Sinai School of Medicine, New York, United States), David H. Miller, MD (University College London, London, United Kingdom), Xavier Montalban, MD (Hospital Universitari Vall d'Hebron, Barcelona, Spain), Paul O'Connor, MD (St Michael's Hospital, Toronto, Canada), Daniel Pelletier, MD (Yale University School of Medicine, New Haven, United States), Stephen C. Reingold, PhD (Scientific \& Clinical Review Assoc, LLC, Salisbury, United States), Alex Rovira Cañellas, MD (Hospital Universitari Vall d'Hebron, Barcelona, Spain), Per Soelberg Sørensen, MD, DMSc (Copenhagen University Hospital, Copenhagen, Denmark), Maria Pia Sormani, $\mathrm{PhD}$ (University of Genoa, Genoa, Italy), Olaf Stuve, MD, $\mathrm{PhD}$ (University of Texas Health Sciences Center, Dallas, United States), Alan J. Thompson, MD (University College London, London, United Kingdom), Maria Trojano, MD (University of Bari, Bari, Italy), Bernard Uitdehaag, $\mathrm{MD}, \mathrm{PhD}$ (VU 
University Medical Center, Amsterdam, Netherlands), Emmaunelle Waubant, MD, PhD (University of California- San Francisco, San Francisco, United States), and Jerry S. Wolinsky, MD (University of Texas HSC, Houston, United States)

\section{Conflicts of interest}

Ruth Ann Marrie receives research funding from: Canadian Institutes of Health Research, Public Health Agency of Canada, Manitoba Health Research Council, Health Sciences Centre Foundation, Multiple Sclerosis Society of Canada, Multiple Sclerosis Scientific Foundation, Rx \& D Health Research Foundation, and has conducted clinical trials funded by Sanofi-Aventis.

Nadia Reider has nothing to declare.

Jeffrey Cohen reports personal compensation for consulting from EMD Serono, Genentech, Genzyme, Innate Immunotherapeutics, Novartis, and Vaccinex. He receives research support paid to his institution from Biogen Idec, Consortium of MS Centers, US Department of Defense, Genzyme, US National Institutes of Health, National MS Society, Novartis, Receptos, Synthon, Teva, and Vaccinex.

Olaf Stuve is an associate editor of JAMA Neurology, and he serves on the editorial boards of the Multiple Sclerosis Journal, Clinical and Experimental Immunology, and Therapeutic Advances in Neurological Disorders. He has participated in data and safety monitoring committees for Pfizer and Sanofi. Dr Stuve has received grant support from Teva Pharmaceuticals.

Maria Trojano has served on scientific advisory boards for Biogen Idec, Novartis, and Merck Serono; has received speaker honoraria from Biogen-Idec, Sanofi Aventis, Merck-Serono, Teva, and Novartis; and has received research grants from Biogen-Idec, Merck-Serono, and Novartis.

Gary Cutter has served on scientific advisory boards for and/or received funding for travel from Innate immunity, Klein-Buendel Incorporated, Genzyme, Medimmune, Novartis, Nuron Biotech, Spiniflex Pharmaceuticals, Somahlution, Teva Pharmaceuticals; receives royalties from publishing Evaluation of Health Promotion and Disease Prevention (The McGraw Hill Companies, 1984); has received honoraria from GlaxoSmithKline, Novartis, Advanced Health Media Inc, Biogen Idec, EMD Serono Inc, EDJ Associates Inc, the National Heart, Lung, and Blood Institute, National Institute of Neurological Diseases and Stroke, National Marrow Donor Program, Consortium of Multiple Sclerosis Centers; Mt. Sinai School of Medicine and Teva Pharmaceuticals; and has served on independent data and safety monitoring committees for Apotek, Ascendis, Biogen-Idec, Cleveland Clinic, Glaxo Smith Klein Pharmaceuticals, Gilead Pharmaceuticals, Modigenetech/Prolor, Merck/ Ono Pharmaceuticals, Merck, Neuren, PCT Bio, Teva, Vivus, NHLBI (Protocol Review Committee), NINDS, NMSS, NICHD (OPRU oversight committee).

Stephen Reingold reports personal consulting fees from the National Multiple Sclerosis Society (NMSS) and the European Committee for Treatment and Research in Multiple Sclerosis (ECTRIMS), during the conduct of this work; and over the past three years, personal consulting fees from Bayer HealthCare, Biogen Idec, Coronado Biosciences Inc, the Cleveland Clinic Foundation, Eli Lilly \& Company, from EMD Serono and Merck Serono, Genentech, F. Hoffmann-LaRoche, Ironwood Pharmaceuticals Inc, ISIS Pharmaceuticals Inc, Medimmune Inc, Novartis Pharmaceuticals Corporation, Observatoire Français de la Sclérosis en Plaques, Opexa Therapeutics, Sanofi-Aventis, SK Biopharmaceuticals, Synthon Pharmaceuticals Inc, Teva Pharmaceutical Industries, and Fondation pour l'aide à la Recherche sur la Sclérosis en Plaques, for activities outside of the submitted work.

Per Soelberg Sorensen has received personal compensation for serving on scientific advisory boards, steering committees, independent data monitoring boards in clinical trials, or speaking at scientific meetings from Biogen Idec, Merck Serono, Novartis, Genmab, Teva, GSK, Genzyme, Bayer Schering, Sanofi-Aventis and MedDay Pharmaceuticals. His research unit has received research support from Biogen Idec, Merck Serono, Teva, Sanofi-Aventis, Novartis, RoFAR, Roche, and Genzyme.

\section{Funding}

This work was supported (in part) by the National Multiple Sclerosis Society and a Don Paty Career Development Award from the MS Society of Canada.

\section{References}

1. Langer-Gould A, Popat RA, Huang SM, et al. Clinical and demographic predictors of long-term disability in patients with relapsing-remitting multiple sclerosis: A systematic review. Arch Neurol 2006; 63: 1686-1691.

2. Marrie RA, Rudick R, Horwitz R, et al. Vascular comorbidity is associated with more rapid disability progression in multiple sclerosis. Neurology 2010; 74 : 1041-1047.

3. Weinstock-Guttman B, Zivadinov R, Horakova D, et al. Lipid profiles are associated with lesion formation over 24 months in interferon- $\beta$ treated 
patients following the first demyelinating event. $J$ Neurol Neurosurg Psychiatry 2013; 84: 1186-1191.

4. Warren SA, Turpin KV, Pohar SL, et al. Comorbidity and health-related quality of life in people with multiple sclerosis. Int J MS Care 2009; 11: 6-16.

5. Christiansen CF, Christensen S, Farkas DK, et al. Risk of arterial cardiovascular diseases in patients with multiple sclerosis: A population-based cohort study. Neuroepidemiology 2010; 35: 267-274.

6. Fleming ST. Multiple sclerosis as a comorbidity: A study of resource utilization and outcomes of care. Clin Perform Qual Health Care 1995; 3: 23-30.

7. Allen NB, Lichtman JH, Cohen HW, et al. Vascular disease among hospitalized multiple sclerosis patients. Neuroepidemiology 2008; 30: 234-238.

8. Kang JH, Chen YH and Lin HC. Comorbidities amongst patients with multiple sclerosis: A population-based controlled study. Eur J Neurol 2010; 17: 1215-1219.

9. Khan F, Pallant J and Brand C. Caregiver strain and factors associated with caregiver self-efficacy and quality of life in a community cohort with multiple sclerosis. Disabil Rehabil 2007; 29: 1241-1250.

10. Lavela SL, Prohaska TR, Furner S, et al. Chronic diseases in male veterans with multiple sclerosis. Prev Chronic Dis 2012; 9: E55.

11. Fleming ST and Blake RL Jr. Patterns of comorbidity in elderly patients with multiple sclerosis. J Clin Epidemiol 1994; 47: 1127-1132.

12. Marrie R, Reider N, Cohen J, et al. A systematic review of the incidence and prevalence of comorbidity in multiple sclerosis: Overview. 2015; 21(3): 263-281.

13. Neyeloff J, Fuchs S and Moreira L. Meta-analyses and Forest plots using a Microsoft Excel spreadsheet: Step-by-step guide focusing on descriptive data analysis. BMC Res Notes 2012; 5: 52.

14. Cox DR. The continuity correction. Biometrika 1970; 57: 217-219.

15. Barcellos LF, Kamdar BB, Ramsay PP, et al. Clustering of autoimmune diseases in families with a high-risk for multiple sclerosis: A descriptive study. Lancet Neurol 2006; 5: 924-931.

16. Buchanan RJ, Schiffer R, Stuifbergen A, et al. Demographic and disease characteristics of people with multiple sclerosis living in urban and rural areas. Int J MS Care 2006; 8: 89-98.

17. Dallmeijer AJ, Beckerman H, de Groot V, et al. Long-term effect of comorbidity on the course of physical functioning in patients after stroke and with multiple sclerosis. J Rehabil Med 2009; 41: 322-326.
18. De Keyser J. Autoimmunity in multiple sclerosis. Neurology 1988; 38: 371-374.

19. Deretzi G, Kountouras J, Koutlas E, et al. Familial prevalence of autoimmune disorders in multiple sclerosis in Northern Greece. Mult Scler 2010; 16: 1091-1101.

20. Eaton WW, Rose NR, Kalaydjian A, et al. Epidemiology of autoimmune diseases in Denmark. $J$ Autoimmun 2007; 29: 1-9.

21. Edwards LJ and Constantinescu CS. A prospective study of conditions associated with multiple sclerosis in a cohort of 658 consecutive outpatients attending a multiple sclerosis clinic. Mult Scler 2004; 10 : 575-581.

22. Finlayson M, Preissner $\mathrm{K}$ and Cho C. Impact of comorbidity on fatigue management intervention outcomes among people with multiple sclerosis. Int $J$ MS Care 2013; 15: 21-26.

23. Fromont A, Binquet C, Rollot F, et al. Comorbidities at multiple sclerosis diagnosis. J Neurol 2013; 260: 2629-2637.

24. Henderson RD, Bain CJ and Pender MP. The occurrence of autoimmune diseases in patients with multiple sclerosis and their families. J Clin Neurosci 2000; 7: 434-437.

25. Hoppenbrouwers IA, Cortes LM, Aulchenko YS, et al. Familial clustering of multiple sclerosis in a Dutch genetic isolate. Mult Scler 2007; 13: 17-24.

26. Horton M, Rudick RA, Hara-Cleaver C, et al. Validation of a self-report comorbidity questionnaire for multiple sclerosis. Neuroepidemiology 2010; 35: 83-90.

27. Hussein WI and Reddy SS. Prevalence of diabetes in patients with multiple sclerosis. Diabetes Care 2006; 29: 1984-1985.

28. Jadidi E, Mohammadi M and Moradi T. High risk of cardiovascular diseases after diagnosis of multiple sclerosis. Mult Scler 2013; 19: 1336-1340.

29. Kanjwal K, Karabin B, Kanjwal Y, et al. Autonomic dysfunction presenting as postural orthostatic tachycardia syndrome in patients with multiple sclerosis. Int J Med Sci 2010; 7: 62-67.

30. Langer-Gould A, Albers K, Van Den Eeden S, et al. Autoimmune diseases prior to the diagnosis of multiple sclerosis: A population-based case-control study. Mult Scler 2010; 16: 855-861.

31. Laroni A, Calabrese M, Perini P, et al. Multiple sclerosis and autoimmune diseases: Epidemiology and HLA-DR association in North-east Italy. J Neurol 2006; 253: 636-639.

32. Lindegård B. Diseases associated with multiple sclerosis and epilepsy: A population cohort study of 
158,200 middle-aged, urban, native Swedes observed over 10 years (1970-79). Acta Neurol Scand 1985; 71: 267-277.

33. Lu E, Zhao Y, Zhu F, et al. Birth hospitalization in mothers with multiple sclerosis and their newborns. Neurology 2013; 80: 447-452.

34. Marrie RA, Yu B, Leung S, et al. Rising prevalence of vascular comorbidities in MS: Validation of administrative definitions for diabetes, hypertension, hyperlipidemia. Mult Scler 2012; 18: 1310-1319.

35. Marrie RA, Horwitz R, Cutter G, et al. Comorbidity, socioeconomic status, and multiple sclerosis. Mult Scler 2008; 14: 1091-1098.

36. Marrosu MG, Cocco E, Lai M, et al. Patients with multiple sclerosis and risk of type 1 diabetes mellitus in Sardinia, Italy: A cohort study. Lancet 2002; 359: 1461-1465.

37. Marrosu MG, Motzo C, Murru R, et al. The co-inheritance of type 1 diabetes and multiple sclerosis in Sardinia cannot be explained by genotype variation in the HLA region alone. Hum Mol Genet 2004; 13: 2919-2924.

38. Midgard R, Grønning M, Riise T, et al. Multiple sclerosis and chronic inflammatory diseases. A case-control study. Acta Neurol Scand 1996; 93: 322-328.

39. Nuyen J, Schellevisa FG, Satarianob WA, et al. Comorbidity was associated with neurologic and psychiatric diseases: A general practice-based controlled study. J Clin Epidemiol 2006; 59: 1274 1284 .

40. Paz Soldan MM, Pittock SJ, Weigand SD, et al. Statin therapy and multiple sclerosis disability in a population-based cohort. Mult Scler 2012; 18: 358-363.

41. Pitzalis M, Zavattari P, Murru R, et al. Genetic loci linked to type 1 diabetes and multiple sclerosis families in Sardinia. BMC Med Genet 2008; 9: 3.

42. Ramagopalan SV, Dyment DA, Valdar W, et al. The occurrence of autoimmune disease in Canadian families with multiple sclerosis. Lancet Neurol 2007; 6: 604-610.

43. Seyfert S, Klapps P, Meisel C, et al. Multiple sclerosis and other immunologic diseases. Acta Neurol Scand 1990; 81: 37-42.

44. Sheu JJ and Lin HC. Association between multiple sclerosis and chronic periodontitis: A population-based pilot study. Eur J Neurol 2013; 20: 1053-1059.

45. Tremlett HL, Evans J, Wiles CM, et al. Asthma and multiple sclerosis: An inverse association in a casecontrol general practice population. QJM 2002; 95 : 753-756.
46. Wertman E, Zilber N and Abramsky O. An association between multiple sclerosis and type I diabetes mellitus. J Neurol 1992; 239: 43-45.

47. Wynn DR, Rodriguez M, O'Fallon WM, et al. A reappraisal of the epidemiology of multiple sclerosis in Olmsted County, Minnesota. Neurology 1990; 40: 780-786.

48. Palo J, Duchesne J and Wikstrom J. Malignant diseases among patients with multiple sclerosis. $J$ Neurol 1977; 216: 217-222.

49. Marrie R, Reider N, Cohen J, et al. A systematic review of the incidence and prevalence of autoimmune disease in multiple sclerosis. Mult Scler. 2015; 21(3): 282-293.

50. Marrie RA, Horwitz RI, Cutter G, et al. Association between comorbidity and clinical characteristics of MS. Acta Neurol Scand 2011; 124: 135-141.

51. Sun LM, Lin CL, Chung CJ, et al. Increased breast cancer risk for patients with multiple sclerosis: A nationwide population-based cohort study. Eur $J$ Neurol 2014; 21: 238-244.

52. Füvesi J, Bencsik K, Losonczi E, et al. Factors influencing the health-related quality of life in Hungarian multiple sclerosis patients. $J$ Neurol Sci 2010; 293: 59-64.

53. Holper L, Coenen M, Weise A, et al. Characterization of functioning in multiple sclerosis using the ICF. $J$ Neurol 2010; 257: 103-113.

54. Hopman WM, Coo H, Edgar CM, et al. Factors associated with health-related quality of life in multiple sclerosis. Can J Neurol Sci 2007; 34: 160-166.

55. Lix L, Yogendran M, Shaw S, et al. Population-based data sources for chronic disease surveillance. Chronic Dis Can 2008; 29: 22-30.

56. Marrie RA, Yu BN, Leung S, et al. Prevalence and incidence of ischemic heart disease in multiple sclerosis: A population-based validation study. Mult Scler Relat Disord 2013; 2: 355-361.

57. Krokki O, Bloigu R, Ansakorpi H, et al. Neurological comorbidity and survival in multiple sclerosis. Mult Scler Relat Disord 2013; 3: 72-77.

58. Marrie RA, Cutter G and Tyry T. Substantial adverse association of visual and vascular comorbidities on visual disability in multiple sclerosis. Mult Scler 2011; 17: 1464-1471.

59. Johnston SC, Mendis S and Mathers CD. Global variation in stroke burden and mortality: Estimates from monitoring, surveillance, and modelling. Lancet Neurol 2009; 8: 345-354. 
60. Feigin VL, Lawes CM, Bennett DA, et al. Worldwide stroke incidence and early case fatality reported in 56 population-based studies: A systematic review. Lancet Neurol 2009; 8: 355-369.

61. Moran AE, Forouzanfar MH, Roth GA, et al. The global burden of ischemic heart disease in 1990 and 2010: The Global Burden of Disease 2010 Study. Circulation 2014; 129: 1493-1501.

62. Levy AR, Tamblyn RM, Fitchett D, et al. Coding accuracy of hospital discharge data for elderly survivors of myocardial infarction. Can J Cardiol 1999; 15: 1277-1282.

63 Ranadive SM, Yan H, Weikert M, et al. Vascular dysfunction and physical activity in multiple sclerosis. Med Sci Sports Exerc 2012; 44: 238-243.

64 Crowson CS, Nicola PJ, Kremers HM, et al. How much of the increased incidence of heart failure in rheumatoid arthritis is attributable to traditional cardiovascular risk factors and ischemic heart disease? Arthritis \& Rheumatism 2005; 52 : 3039-3044.

65 Gelfand JM, Neimann AL, Shin DB, Wang X, Margolis DJ, Troxel AB. Risk of Myocardial Infarction in Patients With Psoriasis. JAMA 2006; 296: 1735-1741.

66. Brinkley DM and Gelfand EV. Valvular heart disease: Classic teaching and emerging paradigms. Am J Med 2013; 126: 1035-1042.

67. Lalmohamed A, Bazelier MT, Van Staa TP, et al. Causes of death in patients with multiple sclerosis and matched referent subjects: A population-based cohort study. Eur J Neurol 2012; 19: 1007-1014.

68. Chataway J, Schuerer N, Alsanousi A, et al. Effect of high-dose simvastatin on brain atrophy and disability in secondary progressive multiple sclerosis (MS-STAT): A randomised, placebocontrolled, phase 2 trial. Lancet 2014; 383: 2213-2221.
Visit SAGE journals online http://msj.sagepub.com

(s) SAGE journals 